\title{
PERSPECTIVE OPEN Force of infection: a determinant of vaccine efficacy?
}

\author{
David C. Kaslow (iD) ${ }^{1 凶}$
}

Vaccine efficacy (VE) can vary in different settings. Of the many proposed setting-dependent determinants of VE, force of infection (Fol) stands out as one of the most direct, proximate, and actionable. As highlighted by the COVID-19 pandemic, modifying Fol through non-pharmaceutical interventions (NPIs) use can significantly contribute to controlling transmission and reducing disease incidence and severity absent highly effective pharmaceutical interventions, such as vaccines. Given that NPIs reduce the Fol, the question arises as to if and to what degree Fol, and by extension NPIs, can modify VE, and more practically, as vaccines become available for a pathogen, whether and which NPIs should continue to be used in conjunction with vaccines to optimize controlling transmission and reducing disease incidence and severity.

npj Vaccines (2021)6:51; https://doi.org/10.1038/s41541-021-00316-5

\section{INTRODUCTION}

Lower apparent vaccine efficacy (VE) in low resource settings, when compared to VE observed in high resource settings, has been reported for several pathogens, most notably poliovirus, typhoid, and rotavirus ${ }^{1-5}$. Observed VE also varied when evaluating a malaria vaccine candidate in different parasite transmission settings ${ }^{6-8}$. Numerous economic, social, and biological factors have been proposed to explain these settingdependent variations in $\mathrm{VE}^{3,9-11}$. Many, if not most, of the proposed economic and social determinants of VE, such as, country income status, living conditions, access to healthcare, appear to act indirectly and non-specifically on VE; whereas many but not all biological factors, such as co-infections, malnutrition, and enteropathy, presumably act directly and proximally on VE. More practically, identification of direct and proximal determinants of setting-dependent VE that hold the promise of actionable intervention(s) seem a most urgent need in efforts to enhance and/or sustain VE.

The COVID-19 pandemic has highlighted the contribution of non-pharmaceutical interventions (NPIs) in controlling transmission and reducing disease incidence and severity ${ }^{12}$, particularly in the absence of highly effective pharmaceutical interventions, such as vaccines. NPIs also contribute to controlling other major human diseases, including use of condoms for HIV/AIDS ${ }^{13}$, bed nets for malaria ${ }^{14}$, and hand washing for diarrhea ${ }^{15}$. By reducing the number of (susceptible) individuals effectively contacted by each (infected) person, e.g., through physical barriers, distancing, and masking, NPIs reduce $\lambda$, the force of infection (Fol) (see Box 1, Glossary of Key Terms). As vaccines become available for a pathogen, the question arises as to if and which NPIs should continue to be used, if not prioritized ${ }^{16}$. This then begs the broader useinspired scientific question, as raised previously ${ }^{8}$ : after optimizing the vaccine immunogen, formulation, dose level, and regimen, what remaining determinants of VE are amenable to intervention? More specifically, given the role of NPIs in reducing the Fol, if and to what degree is Fol, and by extension NPIs, a determinant of VE?

\section{Interrogating the potential relationship of Fol and setting- dependent VE}

A two-step approach was taken to interrogate the potential relationship between Fol and VE. The first explored three mathematical scenarios of VE as a function of various Fol settings. The second followed up on the decades-old observations of lower apparent efficacy of oral poliovirus ${ }^{1}$ and oral typhoid vaccines ${ }^{5}$ in low resource settings when compared to high resource settings. This empiric interrogation assessed the correlation between the incidence of disease in the placebo population (as a surrogate of Fol in the study population) and the observed VE in different geographical settings. Recent Phase 3 studies of malaria and rotavirus vaccine candidates across a number of settings, including low and high resource settings ${ }^{6,17}$, provided data for empirically assessing if and how Fol might be a determinant of VE.

Both the thought experiment of setting-dependent VE of a hypothetical vaccine and the retrospective analyses of rotavirus and malaria Phase 3 efficacy results make a multitude of assumptions that limit the robustness and soundness of any conclusions. For simplicity, factors previously shown or hypothesized to influence transmission, susceptibility, VE, and/or Fol, such as, country income status, age, underlying medical conditions, coinfections, access to healthcare, seasonality, NPI use, spreading events, and strain differences across different settings, and preexposure effect were excluded from consideration in both the hypothetical VE or observed VE analyses.

Given these significant limitations in the analyses, the primary goal of the present study was not to provide a definitive answer to the questions of if and to what degree Fol determines VE in different settings. Rather the goal of these analyses was to continue to raise the awareness of the potential impact of Fol on $\mathrm{VE}^{8,18}$, and to prompt prospective studies designed to assess if and how NPIs might reduce Fol and enhance VE when vaccines are introduced and scaled up. Ultimately well-designed studies that directly evaluate the potential relationship of Fol and settingdependent VE will provide the evidence needed for well-informed policy recommendations on the continued use or not of NPIs during vaccine introduction and scale-up.

1PATH, 2201 Westlake Avenue, Suite 200, Seattle, WA, USA. ${ }^{凶}$ email: dkaslow@path.org 


\section{Box 1 Glossary of key terms}

Force of infection: Rate at which susceptible individuals in a population acquire an infectious disease in that population, per unit time. It is also known as the incidence rate or hazard rate $^{36}$

$\lambda_{t}=\frac{c_{e} l_{t}}{N_{t}}$

(see equation 2.13, ref. ${ }^{36}$ ) where $\lambda_{t}$ is the force of infection at time $t, c_{e}$ is the number of individuals effectively contacted by each person per unit time, $l_{t}$ is the number of infected in the population at time $t$, and $N_{t}$ is the number in the population at time $t$.

Efficacy: The direct protection provided by vaccination against a defined clinical endpoint; it excludes any indirect (herd) effect ${ }^{36}$. Vaccine efficacy reflects the relative reduction between the vaccinated and control groups for one or more specific clinical endpoints. Calculations of the relative reduction typically use a hazard ratio, a risk ratio, or most simply, as shown below, an incidence ratio ${ }^{37}$;

$$
\mathrm{VE}=\frac{\mathrm{ARU}-\mathrm{ARV}}{\mathrm{ARU}} \times 100=1-\frac{\frac{I_{V}}{N_{V}}}{\frac{I_{U}}{N_{U}}} \times 100
$$

(see equations, ref. ${ }^{38}$ ) where VE is the vaccine efficacy, ARU is the attack rate in the unvaccinated population, ARV is the attack rate in the vaccinated population, $I_{V}$ is the number of infected in the vaccinated population, $N_{V}$ number in the vaccinated population, $I_{U}$ is the number of infected in the unvaccinated population, and $N_{U}$ is the number in the unvaccinated population.

Herd immunity: The proportion of a population immune to infection or disease $\mathrm{e}^{2,36}$

Herd immunity threshold: The proportion of the population required to be immune in the population for the infection incidence to reach steady state, i.e., the infection level is neither growing nor declining. To eliminate an infection in the population, the proportion of the population that is immune to infection must exceed this threshold value ${ }^{36}$

Indirect (Herd) effect: The reduction in the rate of infection or disease in the unimmunized portion of a population as a result of immunizing a proportion of the population ${ }^{2}$.

\section{Three scenarios of the potential mathematical consequences of Fol on setting-dependent VE}

The potential effects of Fol on the level of VE were explored in three mathematical scenarios: (1) $\mathrm{VE}_{\text {constant, }}$ where VE is independent of Fol; (2) $\mathrm{VE}_{\text {linear, }}$ where $\mathrm{VE}$ decreases linearly as a function of increasing Fol; and, (3) VE $E_{\text {natural }}$ log, where VE decreases logarithmically as a function of increasing Fol. As noted above, multiple simplifying assumptions were made when considering the mathematical consequences of Fol on VE, including homogeneity in the population with respect to a number of factors, such as, pathogen transmission, host susceptibility to infection and disease (be it genetic or acquired), Fol over time in a specific setting, and protective immunity as a result of vaccination across settings.

With these simplifying assumptions in mind, equations that define the three mathematical scenarios (see Box 2, VE as a function of Fol) are shown graphically in Fig. 1, using the example of a hypothetical vaccine that has a maximum VE of $83 \%$ studied under conditions of Fol that vary across two orders of magnitude, from 0.03 to 3.50 infections/person-year. While other more complex mathematical relationships between VE and Fol merit consideration, these three simple equations seemed a reasonable starting point from which to interrogate observed data from Phase 3 VE studies conducted in multiple epidemiological settings.

\section{Empiric evidence of Fol on observed setting-dependent VE}

Results from recent placebo-controlled Phase 3 studies of vaccine candidates for two diverse pathogens, Plasmodium falciparum and rotavirus, provided a database to determine which, if any, of the three mathematical scenarios best explained any settingdependent differences in VE. The selection of malaria and diarrhea
Box 2 Vaccine efficacy as a function of force of infection

The following equations define mathematical relationships between vaccine efficacy (VE) and force of infection (Fol) shown in Fig. 1, when the relationship of VE is: (1) independent of Fol ( $\left.\mathrm{VE}_{\text {constant }}\right)$; (2) linear to Fol ( $\left(\mathrm{VE}_{\text {linear }}\right)$; or (3) logarithmic to Fol ( $\left.\mathrm{VE}_{\text {natural log }}\right)$ :

$\mathrm{VE}_{\text {constant }}: \mathrm{VE}_{\mathrm{S}}=-0 * \boldsymbol{F o l}_{\mathbf{s}}+\mathrm{VE}_{\max }$

$\mathrm{VE}_{\text {linear }}: \mathrm{VE}_{S}=-\left(\frac{\mathrm{Fol}_{\mathbf{S}}-\mathrm{Fol}_{\min }}{\mathrm{Fol}_{\max }-\mathrm{Fol}_{\min }}\right) \times\left(\mathrm{VE}_{\max }-\mathrm{VE}_{\min }\right)+\mathrm{VE}_{\max }$

$\mathrm{VE}_{\text {natural log }}: \mathrm{VE}_{\mathrm{S}}=-\left(\frac{\ln \mathrm{Fol}_{\mathbf{S}}-\ln \mathrm{Fol}_{\min }}{\ln \mathrm{Fol}_{\max }-\ln \mathrm{Fol}_{\min }}\right) \times\left(\mathrm{VE}_{\max }-\mathrm{VE}_{\min }\right)+\mathrm{VE}_{\max }$

Where $V E_{S}$ is the VE in setting $S, V E_{\max }$ is the highest observed $V E$, and $V E_{\min }$ is the lowest observed VE.

And where $\mathrm{Fol}_{\mathrm{S}}$ is the Fol in setting $S, \mathrm{Fol}_{\min }$ is the lowest observed Fol, and Fol $_{\text {max }}$ is the highest observed Fol.

as clinical endpoints provided an opportunity to analyze Fol and VE for both vector-transmitted and fecal-oral-transmitted pathogens, as well as parenterally and orally administered vaccine candidates, respectively. In addition to the assumptions mentioned above, several additional assumptions noted below facilitated the analyses of these multi-setting VE studies of two pathogens.

First and foremost, the analyses of both pathogens assumed that the intent-to-treat (ITT) incidence of the most sensitive definition of the mildest disease endpoint in the youngest age cohort in the placebo arm best served as an internal Phase 3 study surrogate of $\lambda$, the Fol. The validity of this assumption relies upon several other assumptions, including the absence of any significant herd effect (see Box 1, Glossary of Key Terms) on the control from the vaccinated arm of the Phase 3 study. The rationale for making this herd effect assumption, typically also assumed for the control group used in estimating VE in the context of Phase 3 efficacy studies, relies upon: (1) the relatively small proportion of the total population in the study setting enrolled in the vaccinated group in the Phase 3 study; and, (2) the timing of incident disease in the control group relative to eliciting herd immunity and reaching the herd immunity threshold (see Box 1, Glossary of Key Terms) in the study population.

A third key assumption relied upon a comparison of trendlines from the three mathematical scenarios described above to the closest fit trendline of observed $V E\left(V_{\text {observed }}\right)$ as

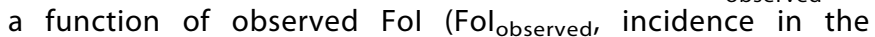
placebo group) in each epidemiologic setting to determine if and how VE varied as a function of Fol. In this regard, because the Phase 3 VE results for both pathogens were known a priori to vary by epidemiologic setting, the posterior probability was low of selecting the $\mathrm{VE}_{\text {constant }}$ mathematical scenario to categorize $\mathrm{VE}_{\text {observed }}$ as a function of $\mathrm{Fol}_{\text {observed. }}$ As noted below for each specific analysis, the observed trendline may not necessarily reflect a statistically significant association between $\mathrm{VE}_{\text {observed }}$ and $\mathrm{Fol}_{\text {observed, }}$ as assessed by a regression analysis.

\section{Malaria parasite VE and Fol}

A single pivotal Phase 3 VE study (NCT00866619) enrolled 15,459 participants in two age categories (young children aged 5-17 months and infants aged 6-12 weeks at the time of enrollment) across 11 clinical research sites in seven African countries (one site in Burkina Faso, Gabon, Malawi, and Mozambique; two sites in Ghana and Tanzania; and three sites in Kenya). The trial assessed, as a primary aim, VE of a three-dose regimen of $\mathrm{RTS}, \mathrm{S} / \mathrm{ASO}_{\mathrm{E}}$ against clinical malaria 


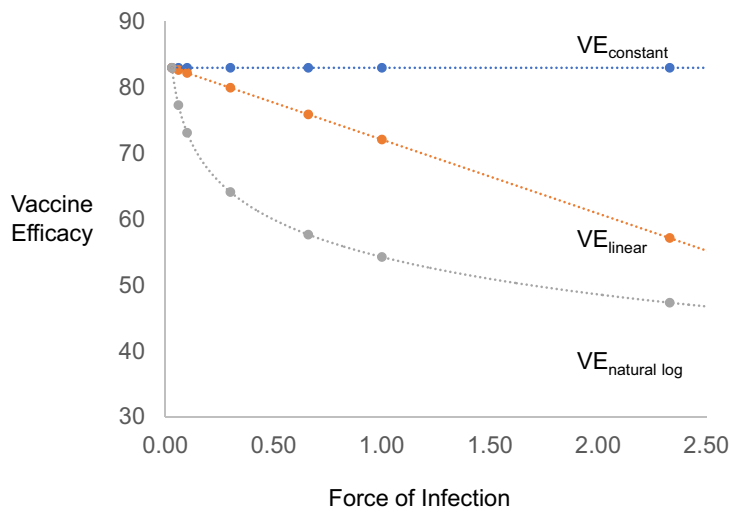

\begin{tabular}{|c|c|c|c|}
\hline \multicolumn{3}{|c|}{$\mathrm{VE}_{\text {max }}: 83.0$} & Fol $_{\text {min }}: 0.03$ \\
$\mathrm{VE}_{\text {min }}: 44.0$ & Fol $_{\text {max }}: 3.50$ \\
\hline $\mathrm{VE}_{\text {constant }}$ & $\mathrm{VE}_{\text {linear }}$ & $\mathrm{VE}_{\text {natural log }}$ & Fol \\
\hline 83.0 & 83.0 & 83.0 & 0.03 \\
\hline 83.0 & 82.7 & 77.3 & 0.06 \\
\hline 83.0 & 82.2 & 73.1 & 0.10 \\
\hline 83.0 & 80.0 & 64.1 & 0.30 \\
\hline 83.0 & 75.9 & 57.7 & 0.66 \\
\hline 83.0 & 72.1 & 54.3 & 1.00 \\
\hline 83.0 & 57.1 & 47.3 & 2.33 \\
\hline 83.0 & 44.0 & 44.0 & 3.50 \\
\hline
\end{tabular}

Force of Infection

Fig. 1 Vaccine Efficacy (VE) as a function of force of Infection (Fol) for hypothetical vaccine. Equations that define three mathematical scenarios (see Box 2, Vaccine efficacy as a function of force of infection) are shown graphically, using as an example a hypothetical vaccine with a maximum vaccine efficacy $\left(V^{\max }\right)$ of $83.0 \%$ and minimum $V E\left(V E_{\min }\right)$ of $44.0 \%$ studied under conditions of force of infection (Fol) that vary across two orders of magnitude, from a minimum Fol ( $\mathrm{Fol}_{\text {min }}$ ) 0.03 to a maximum Fol (Fol max of 3.50 infections/ person-year.

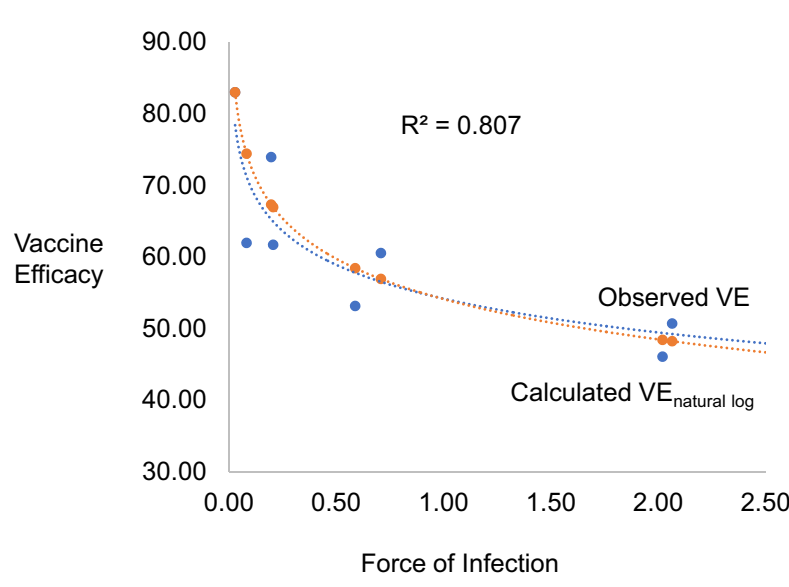

\begin{tabular}{|c|c|c|}
\hline \multicolumn{2}{|c|}{$\begin{array}{l}\mathrm{VE}_{\max }: 83.0 \\
\mathrm{VE}_{\text {min }}: 44.0\end{array}$} & Fol $_{\text {min }}: 0.03$ \\
\hline $\mathrm{VE}_{\text {observed }}$ & $\mathrm{VE}_{\text {matural log }}: 3.46$ & Fol $_{\text {observed }}$ \\
\hline 83.0 & 83.0 & 0.03 \\
\hline 62.0 & 74.4 & 0.08 \\
\hline 74.0 & 67.3 & 0.20 \\
\hline 61.7 & 67.0 & 0.21 \\
\hline 53.2 & 58.4 & 0.59 \\
\hline 60.6 & 56.9 & 0.71 \\
\hline 46.0 & 48.4 & 2.02 \\
\hline 50.7 & 48.2 & 2.06 \\
\hline 44.0 & 45.1 & 3.04 \\
\hline 49.9 & 44.0 & 3.46 \\
\hline
\end{tabular}

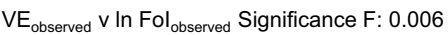

Fig. 2 Vaccine Efficacy (VE) as a function of Force of Infection (Fol) for malaria vaccine. Best fit trendline analysis of observed vaccine efficacy ( $\left(\mathrm{E}_{\text {observed }}\right)$ as a function of observed force of infection (Folobserved) is shown as a logarithmic relationship (blue dotted line) with a $R^{2}$ of 0.807 . A regression analysis of $\mathrm{VE}_{\text {observed }}$ as a function of In Fol observed shown in the embedded table has a Significance $\mathrm{F}$ of 0.006. Using the $\mathrm{VE}_{\text {natural log }}$ equation (see Box 2, Vaccine efficacy as a function of force of infection), the observed $V E_{m a x}, V_{m i n}, F_{m a x}$ $\mathrm{Fol}_{\min }$ and $\mathrm{Fol}_{\text {observed }}$ were used to calculate the $\mathrm{VE}_{\text {natural log }}$ in the embedded table and the calculated $\mathrm{VE}_{\text {natural log }}$ shown graphically (orange dotted line).

over 12 months follow-up ${ }^{7}$. In the per-protocol population of the 5-17 months age category, $\mathrm{VE}_{\text {observed }}$ was $51.3 \%(95 \% \mathrm{Cl}$ : $47.5-54.9 ; p$-value $<.0001$ ) with a $\mathrm{VE}_{\text {observed }}$ range from $83.0 \%$ $(95 \% \mathrm{Cl}: 37.2-95.4 ; p$-value 0.0079$)$ in a low parasite transmission site (Kilifi, Kenya) to $44.0 \%$ (95\% Cl: $36.8-50.3$; $p$-value $<.0001$ ) in a high parasite transmission site (Nanoro, Burkina Faso) (see Annex 6 Table 23, ref. ${ }^{19}$ ). As noted above, the intent-to-treat (ITT) incidence of the more sensitive secondary definition of clinical malaria in the control group of infants aged 6-12 weeks at the time of enrollment (see Annex 7 Table

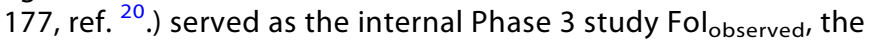
surrogate of $\lambda$ in the analyses.

The best fit trendline analysis of $\mathrm{VE}_{\text {observed }}$ as a function of Fol $_{\text {observed }}$ revealed a logarithmic relationship (Fig. 2, Observed $\mathrm{VE})$ with an $R^{2}$ of 0.807 . Regression analysis of $\mathrm{VE}_{\text {observed as a }}$

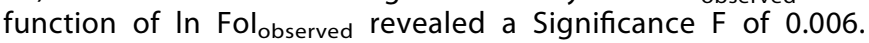
Using the $V E_{\text {natural }}$ log equation (Box 2), the observed $V E_{\max }$, $\mathrm{VE}_{\min }, \mathrm{Fol}_{\max }, \mathrm{Fol}_{\min }$ and the $\mathrm{Fol}_{\text {observed }}$ from each site generated a logarithmic relationship between the calculated

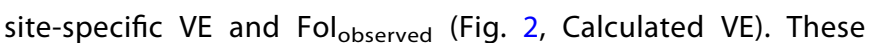
analyses suggest that malaria parasite Fol functions as a determinant of RTS,S/ASO1 $\mathrm{E}$ V.

\section{Rotavirus VE and Fol}

Multiple Phase 3 studies of two rotavirus vaccines, RV1 (Rotarix ${ }^{\oplus}$ ) and RV5 (RotaTeq ${ }^{\oplus}$ ), evaluated VE in diverse epidemiologic settings ${ }^{17}$. In comparison to the analyses conducted for malaria $V E$, the analyses of rotavirus $V_{\text {observed }}$ as a function of rotavirus $\mathrm{Fol}_{\text {observed }}$ was complicated by the evaluation of two different vaccine candidates, with two different regimens, in several different clinical protocols. Some of the Phase 3 studies conducted in low resource settings did not collect data on the incidence of rotavirus gastroenteritis (RVGE) of any severity. The analyses excluded these studies due to the absence of an intent-to-treat incidence of any severity RVGE in the placebo group to serve as a surrogate of $\lambda$. The analyses also excluded data from countries in which the placebo group 
Table 1. Rotavirus vaccine Phase 3 study settings by country, World Bank country income classification, and surrogate observed force of infection.

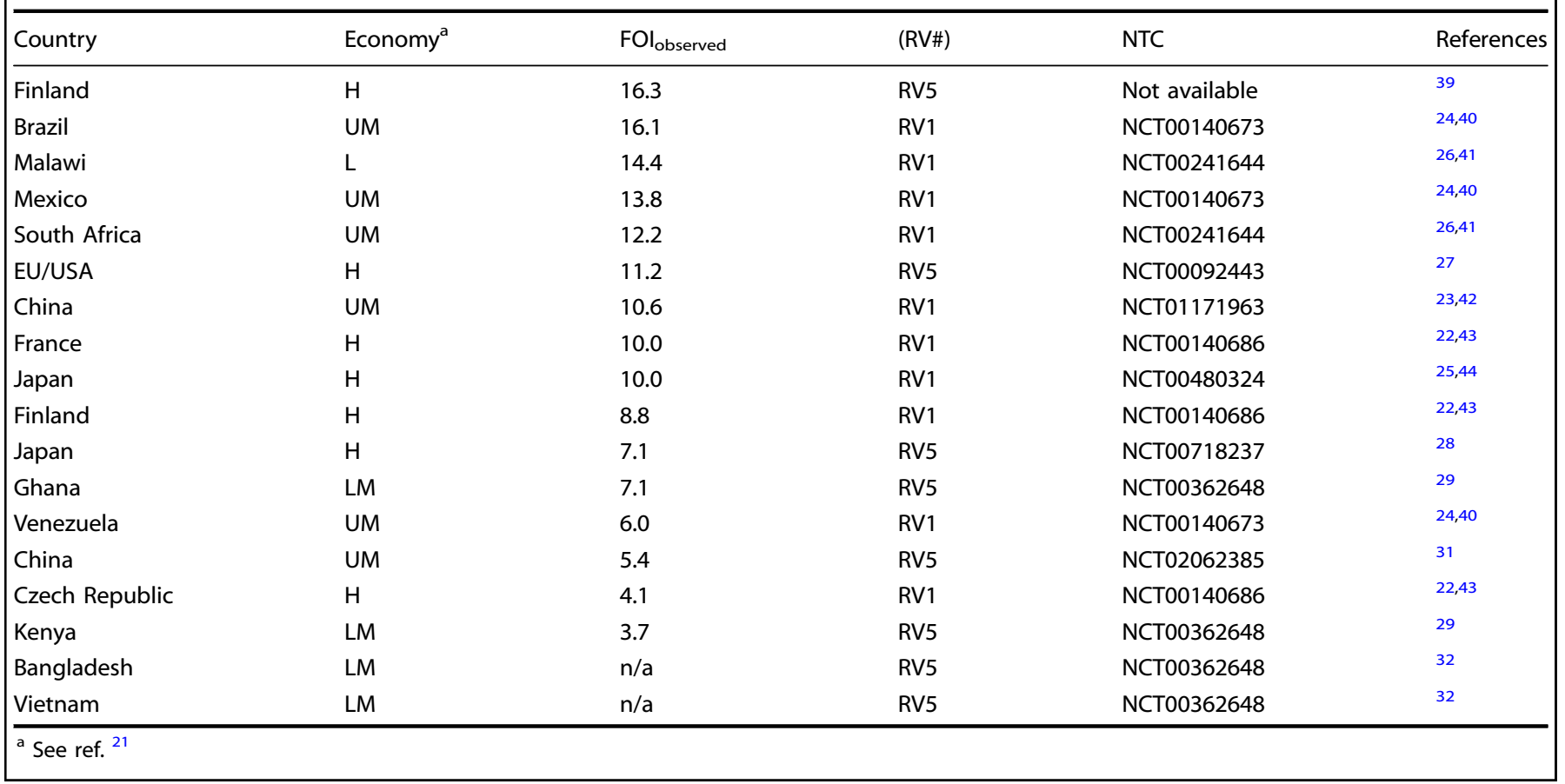

had no or just a single case of RVGE of any severity. From those studies that collected sufficient incidence of any severity RVGE in the placebo group, an Analysis of Variance failed to detect a statistically significant difference $(p$-value $=0.749)$ when categorizing Fol observed by 2020 World Bank country income classifications (i.e., upper- $v$ upper middle- $v$ lower middle/ lower-income country) ${ }^{21}$ (Table 1).

For RV1, results from 10 countries in five independent Phase 3 studies $^{17,22-26}$ (see Table 1) met the above Fol observed criteria for interrogation. The best fit trendline analysis of $\mathrm{VE}_{\text {observed }}$ as a function of $\mathrm{Fol}_{\text {observed }}$ revealed a linear relationship (Fig. 3a upper line, Observed VE) with an $R^{2}$ of 0.3892 and regression analysis with a Significance $F$ of 0.158 . The $\mathrm{VE}_{\text {observed }}$ of $94.9 \%$ in one setting (Mexico) with Fol observed of 13.79 appeared to be a significant outlier. Reanalysis absent the data from Mexico revealed a linear relationship (Fig. 3a middle line, Observed VE), with an $R^{2}$ of 0.6264 and regression analysis Significance $F$ of 0.0449. Using the $\mathrm{VE}_{\text {linear }}$ equation (Box 2), the observed $\mathrm{VE}_{\max }$,

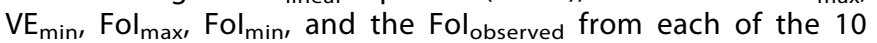
countries generated a linear relationship between the calculated site-specific VE and Fol observed (Fig. 3a lower line, Calculated VE). These analyses suggest that rotavirus Fol may function as a determinant of RV1 VE.

For RV5, results from five settings in three independent Phase 3 studies $^{17,27-31}$ (see Table 1) met the above Fol ${ }_{\text {observed }}$ criteria for interrogation. The best fit trendline analysis of $\mathrm{VE}_{\text {observed }}$ as a function of $\mathrm{Fol}_{\text {observed }}$ revealed an independent relationship (data not shown but provided for review) with an $R^{2}$ of -0.215 and regression analysis Significance $\mathrm{F}$ of 0.9838 . Interrogating results from 7 settings in five independent Phase 3 studies $^{17,27-32}$ (see Table 1) by using the incidence of SRVGE in the placebo group as the Fol observed and surrogate of $\lambda$ in the analyses, the best fit trendline analysis of $\mathrm{VE}_{\text {observed }}$ as a function of Fol $_{\text {observed }}$ revealed a linear relationship (Fig. 3b, Observed VE) with an $R^{2}$ of 0.6692 and regression analysis Significance $F$ of 0.081 . Using the $\mathrm{VE}_{\text {linear }}$ equation (Box 2), the observed $\mathrm{VE}_{\max }, \mathrm{VE}_{\min }$, $\mathrm{Fol}_{\max }, \mathrm{Fol}_{\min }$, and the Fol observed from each of the 7 settings in the reanalysis generated a linear relationship between the calculated site-specific VE and

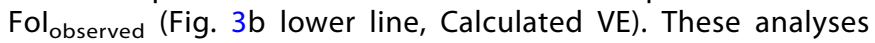
suggest that rotavirus Fol may function as a determinant of RV 5 $V E$, when the incidence of SRVGE, rather than RVGE of any severity, in the placebo group serves as the Fol observed in the analyses.

\section{CONCLUSION}

That a relationship between Fol and VE appears logarithmic for a parenterally administered malaria vaccine candidate and linear for two orally administered rotavirus vaccine candidates may reflect different routes of infection, routes of vaccine administration, fold differences between the $\mathrm{Fol}_{\max }$ and $\mathrm{Fol}_{\min }$ (i.e., more than a hundred-fold for malaria and less than tenfold for rotavirus) or other differences between the pathogens, host responses, or vaccines. If a causal relationship rather than an indirect (e.g., pre-exposure effect ${ }^{33}$ ), misleading ${ }^{34}$, or chance association between Fol and VE exists, then of the many proposed determinants of setting-dependent VE, Fol provides one of the most direct, mechanistically proximate potential determinants. Furthermore, for many but not all pathogens, modifying the Fol provides one of the most actionable interventions to enhance or sustain VE. While improving indirect or distal VE determinants, such as poverty, gut pathology, co-infections, malnutrition, and the microbiome $^{35}$ could significantly enhance efforts to control and eliminate simultaneously many pathogens, implementing interventions that effectively mitigate these VE determinants is complex and not immediately achievable. In contrast, modifying the Fol through the concomitant use of affordable, accessible, available, acceptable, and sustainable NPIs provides a proximate and actionable approach to optimizing VE. Considering and then prospectively verifying the speculation that introduction or continued optimal use of NPIs in an effort to reduce the Fol and thereby enhance or sustain VE, 


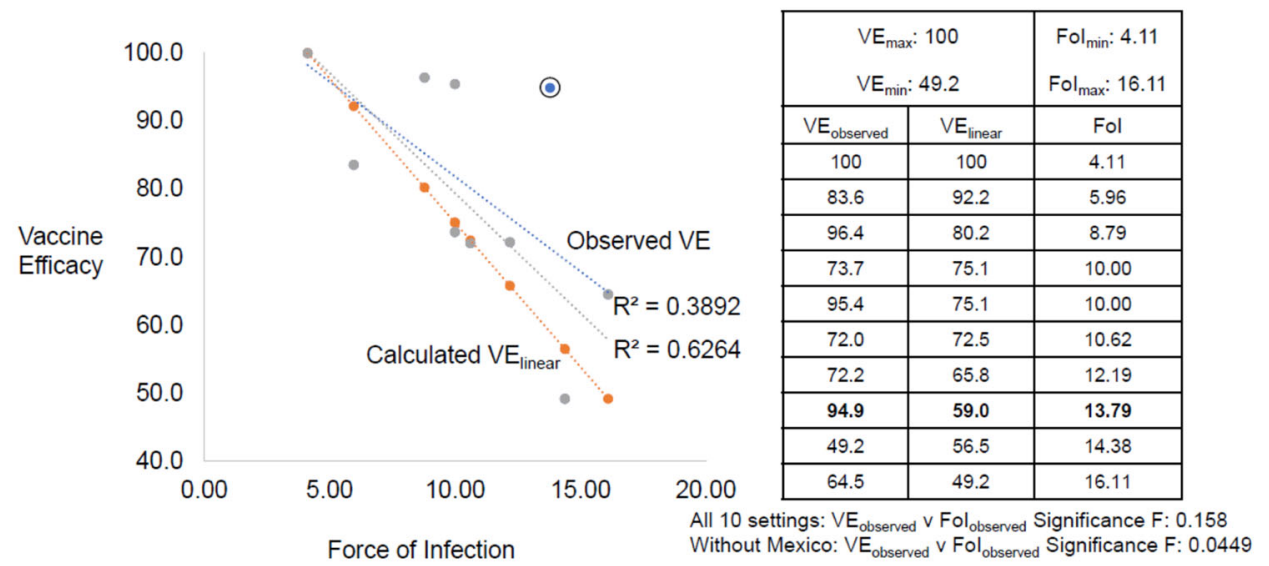

VE SVRGE v SRVGE Fol (RV5)

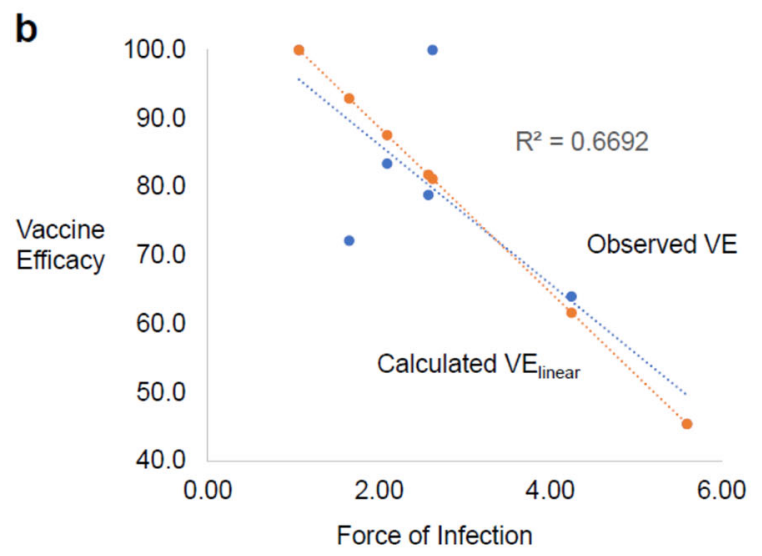

\begin{tabular}{|c|c|c|}
\hline \multicolumn{2}{|c|}{$\begin{array}{c}V^{2} E_{\text {max }}: 100 \\
V E_{\text {min }}: 45.4\end{array}$} & $\begin{array}{c}\text { Fol }_{\text {min }}: 1.06 \\
\text { Fol }_{\text {max }}: 5.60\end{array}$ \\
\hline$V_{\text {observed }}$ & $V E_{\text {linear }}$ & Fol \\
\hline 100 & 100 & 1.06 \\
\hline 72.2 & 92.9 & 1.65 \\
\hline 83.4 & 87.6 & 2.09 \\
\hline 78.8 & 81.8 & 2.58 \\
\hline 100 & 81.2 & 2.62 \\
\hline 64.0 & 61.6 & 4.25 \\
\hline 45.4 & 45.4 & 5.60 \\
\hline
\end{tabular}

Fig. 3 Vaccine Efficacy (VE) as a function of Force of Infection (Fol) for rotavirus vaccines. a RV1: Best fit trendline analysis of observed vaccine efficacy $\left(V_{E_{\text {observed }}}\right)$ as a function of observed force of infection (Fol ${ }_{\text {observed }}$ ) is shown as a linear relationship for all 10 countries (blue dotted line) and for 9 countries (exclusion of the outlier, encircled blue dot; gray dotted line) with a $R^{2}$ of 0.3892 and 0.6264 , respectively.

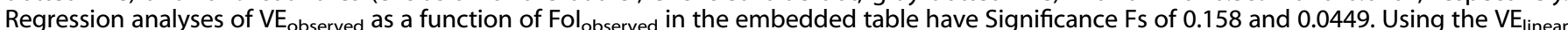
equation (see Box 2, Vaccine efficacy as a function of force of infection), the observed $\mathrm{VE}_{\max }, \mathrm{VE}_{\min }, \mathrm{Fol}_{\max }, \mathrm{FOI}_{\min }$ and Fol ${ }_{\mathrm{observed}} \mathrm{Were}$ used to calculate the $\mathrm{VE}_{\text {linear }}$ in the embedded table and the calculated $\mathrm{VE}_{\text {linear }}$ shown graphically (orange dotted line). $\mathbf{b}$ RV5: Best fit trendline analysis of observed vaccine efficacy ( $\mathrm{VE}_{\text {observed }}$ ) as a function of observed force of infection ( Fol $_{\text {observed }}$ ) is shown as a linear relationship (blue dotted line) with a $R^{2}$ of 0.6692 . A regression analysis of $\mathrm{VE}_{\text {observed }}$ as a function of Fol observed in the embedded table has a Significance $\mathrm{F}$ of 0.081 . Using the $\mathrm{VE}_{\text {linear }}$ equation (see Box 2, Vaccine efficacy as a function of force of infection), the observed $\mathrm{VE}_{\max }, \mathrm{VE}_{\min }, \mathrm{Fol}_{\max }, \mathrm{FOI}_{\min }$ and $\mathrm{Fol}_{\text {observed }}$ were used to calculate the $\mathrm{VE}_{\text {linear }}$ in the embedded table and the calculated $\mathrm{VE}_{\text {linear }}$ shown graphically (orange dotted line).

respectively, upon vaccine rollout seems prudent and, in the context of a pandemic, quite urgent.

\section{Reporting summary}

Further information on research design is available in the Nature Research Reporting Summary linked to this article.

\section{DATA AVAILABILITY}

All data used in the analyses can be publicly accessed. The sources and web links for all the data have been cited in the references.

\section{CODE AVAILABILITY}

All analyses conducted using Microsoft ${ }^{\circledR}$ Excel $^{\circledR}$ for Microsoft 365 version 2101.
Received: 21 January 2021; Accepted: 12 March 2021;

Published online: 12 April 2021

\section{REFERENCES}

1. John, T. J. Immunisation Against polioviruses in developing countries. Rev. Med. Virol. 3, 149-160 (1993).

2. John, T. J. \& Samuel, R. Herd immunity and herd effect: new insights and definitions. Eur. J. Epidemiol. 16, 601-606 (2000).

3. Desselberger, U. Differences of rotavirus vaccine effectiveness by country: likely causes and contributing factors. Pathogens 6, 65 (2017).

4. Lamberti, L. M., Ashraf, S., Walker, C. L. F. \& Black, R. E. A systematic review of the effect of rotavirus vaccination on diarrhea outcomes among children younger than 5 years. Pediatr. Infect. Dis. J. 35, 992-998 (2016).

5. Simanjuntak, C. H. et al. Oral immunisation against typhoid fever in Indonesia with Ty21a vaccine. Lancet Lond. Engl. 338, 1055-1059 (1991). 
6. EMA. Mosquirix: Public Assessment Report. Mosquirix H-W-2300. https://www.ema. europa.eu/en/documents/medicine-outside-eu/mosquirix-public-assessmentreport_en. pdf (2015)

7. Kaslow, D. C. \& Biernaux, S. RTS,S: toward a first landmark on the malaria vaccine technology roadmap. Vaccine 33, 7425-7432 (2015).

8. Kaslow, D. C. Certainty of success: three critical parameters in coronavirus vaccine development. npj Vaccines 5, 1-7 (2020).

9. Fischer Walker, C. L. \& Black, R. E. Rotavirus vaccine and diarrhea mortality: quantifying regional variation in effect size. BMC Public Health 11, S16 (2011).

10. Gruber, J. F. et al. Heterogeneity of rotavirus vaccine efficacy among infants in developing countries. Pediatr. Infect. Dis. J. 36, 72-78 (2017)

11. Jiang, V., Jiang, B., Tate, J., Parashar, U. D. \& Patel, M. M. Performance of rotavirus vaccines in developed and developing countries. Hum. Vaccin. 6, 532-542 (2010).

12. Chu, D. K. et al. Physical distancing, face masks, and eye protection to prevent person-to-person transmission of SARS-CoV-2 and COVID-19: a systematic review and meta-analysis. Lancet 395, 1973-1987 (2020).

13. Weller, S. C. \& Davis-Beaty, K. Condom effectiveness in reducing heterosexual HIV transmission. Cochrane Database Syst. Rev. https://doi.org/10.1002/14651858. CD003255 (2002).

14. Pryce, J., Richardson, M. \& Lengeler, C. Insecticide-treated nets for preventing malaria. Cochrane Database Syst. Rev. 11, CD000363 (2018).

15. Ejemot-Nwadiaro, R. I., Ehiri, J. E., Arikpo, D., Meremikwu, M. M. \& Critchley, J. A. Hand-washing promotion for preventing diarrhoea. Cochrane Database Syst. Rev. https://doi.org/10.1002/14651858.CD004265.pub4 (2020).

16. Winskill, P., Walker, P. G., Griffin, J. T. \& Ghani, A. C. Modelling the costeffectiveness of introducing the RTS,S malaria vaccine relative to scaling up other malaria interventions in sub-Saharan Africa. BMJ Glob. Health 2, e000090 (2017).

17. Soares-Weiser, K., Bergman, H., Henschke, N., Pitan, F. \& Cunliffe, N. Vaccines for preventing rotavirus diarrhoea: vaccines in use. Cochrane Database Syst. Rev. https://doi.org/10.1002/14651858.CD008521.pub5 (2019).

18. Feikin, D. R., Scott, J. A. G. \& Gessner, B. D. Use of vaccines as probes to define disease burden. Lancet 383, 1762-1770 (2014).

19. GSK. Annex 6 to the Clinical Study Report for Study 110021 (MALARIA-055 PRI). https://s3.amazonaws.com/ctr-gsk-7381/110021/640ed3e6-81da-4067-90dbbc8324f71a29/8946951f-0cfe-4ff7-b0e9-1103468776dc/gsk-110021-clinicalstudy-report-redact-01-v1-v3.pdf.

20. GSK. Annex 7 to the Clinical Study Report for Study 110021 (MALARIA-055 PRI). https://s3.amazonaws.com/ctr-gsk-7381/110021/9e419df1-fb51-4b82-8db24256fe3ae230/976c7711-02eb-405b-817f-859bcdb779e2/gsk-110021-clinicalstudy-report-redact-02-v1-v3.pdf.

21. World Bank Country and Lending Groups-World Bank Data Help Desk. https:// datahelpdesk.worldbank.org/knowledgebase/articles/906519-world-bankcountry-and-lending-groups.

22. Vesikari, T. et al. Efficacy of human rotavirus vaccine against rotavirus gastroenteritis during the first 2 years of life in European infants: randomised, doubleblind controlled study. Lancet 370, 1757-1763 (2007).

23. Li, R. et al. Human rotavirus vaccine (RIX4414) efficacy in the first two years of life. Hum. Vaccines Immunother. 10, 11-18 (2014).

24. Salinas, B. et al. Evaluation of safety, immunogenicity and efficacy of an attenuated rotavirus vaccine, rix4414: a randomized, placebo-controlled trial in latin American infants. Pediatr. Infect. Dis. J. 24, 807-816 (2005)

25. Kawamura, N. et al. Efficacy, safety and immunogenicity of RIX4414 in Japanese infants during the first two years of life. Vaccine 29, 6335-6341 (2011).

26. Madhi, S. A. et al. Effect of human rotavirus vaccine on severe diarrhea in African infants. N. Engl. J. Med. 362, 289-298 (2010).

27. Block, S. L. et al. Efficacy, immunogenicity, and safety of a pentavalent humanbovine (WC3) reassortant rotavirus vaccine at the end of shelf life. Pediatrics 119, 11-18 (2007).

28. Iwata, S. et al. Efficacy and safety of pentavalent rotavirus vaccine in Japan. Hum. Vaccines Immunother. 9, 1626-1633 (2013).

29. Armah, G. E. et al. Efficacy of pentavalent rotavirus vaccine against severe rotavirus gastroenteritis in infants in developing countries in sub-Saharan Africa: a randomised, double-blind, placebo-controlled trial. Lancet 376, 606-614 (2010).

30. Vesikari, T. et al. Safety and efficacy of a pentavalent human-bovine (WC3) reassortant rotavirus vaccine. N. Engl. J. Med. 354, 23-33 (2006).

31. EMA. Assessment report for paediatric studies submitted according to Article 46 of the Regulation (EC) No 1901/2006. https://www.ema.europa.eu/en/documents/ variation-report/rotateq-h-c-669-p46-0045-epar-assessment-report en.pdf (2006).

32. Zaman, K. et al. Efficacy of pentavalent rotavirus vaccine against severe rotavirus gastroenteritis in infants in developing countries in Asia: a randomised, double-blind, placebo-controlled trial. Lancet Lond. Engl. 376, 615-623 (2010).
33. O’Hagan, J. J., Lipsitch, M. \& Hernán, M. A. Estimating the per-exposure effect of infectious disease interventions. Epidemiology 25, 134-138 (2014).

34. Lewnard, J. A., Tedijanto, C., Cowling, B. J. \& Lipsitch, M. Measurement of vaccine direct effects under the test-negative design. Am. J. Epidemiol. 187, 2686-2697 (2018).

35. Zimmermann, P. \& Curtis, N. The influence of the intestinal microbiome on vaccine responses. Vaccine 36, 4433-4439 (2018).

36. Vynnycky, E. \& White, R. An Introduction to Infectious Disease Modelling (Oxford University Press, 2010).

37. Halloran, M. E., Longini, I. M. \& Struchiner, C. J. (eds) In Design and Analysis of Vaccine Studies. 103-129 (Springer New York, 2010).

38. Orenstein, W. A. et al. Field evaluation of vaccine efficacy. Bull. World Health Organ 63, 1055-1068 (1985).

39. Vesikari, T. et al. Effects of the potency and composition of the multivalent human-bovine (WC3) reassortant rotavirus vaccine on efficacy, safety and immunogenicity in healthy infants. Vaccine 24, 4821-4829 (2006).

40. GSK. Evaluate protective efficacy of diff strengths of human rotavirus vaccine after administration of 2 doses to infants aged 2 months: GSK 444563-006 Clinical Study Report. https://filehosting-v2.pharmacm.com/api/Attachment/ Download?tenantld $=80217381$ \&parentldentifier $=444563 / 006 \&$ attachmentlde ntifier $=$ ff878ec8-e42b-49cb-b9ef-6ea90ea32e86\&fileName =gsk-444563-006clinical-study-report-redact.pdf\&versionldentifier (2003).

41. GSK. Vaccine Efficacy against Rotavirus Diarrhea; Vaccine Given with Routine Childhood Vaccinations in Healthy African Infants: GSK 102248 Clinical Study Report. https://filehosting-v2.pharmacm.com/api/Attachment/Download?tenantld=80217381 \&parentldentifier $=102248 \&$ attachmentldentifier $=51$ cebf27-e59a-4c45-bed7-154 29582d324\&fileName=gsk-102248-clinical-study-report-redact.pdf\&version dentifier (2009).

42. GSK. Study to Assess the Efficacy, Immunogenicity and Safety of Liquid Human Rotavirus Vaccine, in Healthy Chinese Infants: GSK-113808-Clinical Study Report. https://filehosting-v2.pharmacm.com/api/Attachment/Download?tenantld=8021 7381\&parentldentifier $=113808$ \&attachmentldentifier $=5056 \mathrm{~b} 56 \mathrm{~b}-5311-466 \mathrm{e}-9 \mathrm{f} 63$ d1 b647b37b89\&fileName=gsk-113808-clinical-study-report-redact.pdf\&versionl dentifier (2015).

43. GSK. To test 2 doses of GSK Biologicals' oral live attenuated human rotavirus (HRV) vaccine in healthy infants in co-administration with specific childhood vaccines: GSK 102247/036 Clinical Study Report. https://filehosting-v2.pharmacm.com/api/ Attachment/Download?tenantld $=80217381 \&$ parentldentifier $=102247 / 036 \&$ attach mentldentifier $=$ d31 bed4a-8976-4b17-adf5-aba92145c9ca\&fileName = gsk-102247036-c-clinical-study-report-redact.pdf\&versionldentifier (2006).

44. GSK. Efficacy, safety, reactogenicity \& immunogenicity of the Rotarix vaccine in Japanese infants: GSK 107625 Clinical Study Report. https://filehosting-v2.pharmacm. com/api/Attachment/Download?tenantld=80217381\&parentldentifier $=107625 \&$ attachmentldentifier $=4$ b357e3f-f6a9-467a-ab54-0dc4f0a63da7\&fileName $=$ gsk 107625-clinical-study-report-redact.pdf\&versionldentifier (2009).

\section{ACKNOWLEDGEMENTS}

The author thanks Dr. Marc Lipsitch for critical review and suggestions. This work was supported by the Bill \& Melinda Gates Foundation, Seattle, WA [OPP1180199]. The funder had no role in the preparation of the manuscript or decision to publish.

\section{AUTHOR CONTRIBUTIONS}

D.C.K. conceived, wrote, reviewed, approved and is accountable for this paper.

\section{COMPETING INTERESTS}

D.C.K., an employee of PATH (a not-for-profit organization), has no financial interest in any for-profit organization, and declares no competing interests.

\section{ADDITIONAL INFORMATION}

Supplementary information The online version contains supplementary material available at https://doi.org/10.1038/s41541-021-00316-5.

Correspondence and requests for materials should be addressed to D.C.K.

Reprints and permission information is available at http://www.nature.com/ reprints

Publisher's note Springer Nature remains neutral with regard to jurisdictional claims in published maps and institutional affiliations. 
Open Access This article is licensed under a Creative Commons Ac Attribution 4.0 International License, which permits use, sharing,
adaptation, distribution and reproduction in any medium or format, as long as you give appropriate credit to the original author(s) and the source, provide a link to the Creative Commons license, and indicate if changes were made. The images or other third party material in this article are included in the article's Creative Commons license, unless indicated otherwise in a credit line to the material. If material is not included in the article's Creative Commons license and your intended use is not permitted by statutory regulation or exceeds the permitted use, you will need to obtain permission directly from the copyright holder. To view a copy of this license, visit http://creativecommons. org/licenses/by/4.0/.

(c) The Author(s) 2021 